\title{
Role of transvaginal ultrasonographic cervical assessment in predicting the outcome of induction of labor
}

\author{
Kiran Aggarwal ${ }^{1}$, Anita Yadav ${ }^{2 *}$
}

\begin{abstract}
${ }^{1}$ Department of Obstetrics and Gynecology, Lady Hardinge Medical College, New Delhi, Delhi, India
${ }^{2}$ Department of Obstetrics and Gynecology, Andaman and Nicobar Islands Institute of Medical Sciences, Port Blair, Andaman and Nicobar Islands, India
\end{abstract}

Received: 10 December 2018

Accepted: 07 January 2019

\author{
*Correspondence: \\ Dr. Anita Yadav, \\ E-mail: yadavdr.anita@gmail.com
}

Copyright: ( $\odot$ the author(s), publisher and licensee Medip Academy. This is an open-access article distributed under the terms of the Creative Commons Attribution Non-Commercial License, which permits unrestricted non-commercial use, distribution, and reproduction in any medium, provided the original work is properly cited.

\begin{abstract}
Background: Induction of labor is the most common intervention in modern obstetrics. The pre-induction 'favourability' of the cervix as assessed by the bishop score is very subjective. Transvaginal sonography appears to be a feasible alternative to the traditional bishop's score. Aim of this study, was to compare cervical assessment by transvaginal sonography and digital examination in prediction of outcome of labor induction.

Methods: Three hundred women at term with maternal and foetal indications for labor induction were included in the study. Modified Bishop score was assessed by digital examination and the cervical length was measured by Transvaginal sonography (TVS). Successful labor induction was taken as a vaginal delivery within 24 hours from the start of induction. Data were analyzed using SPSS for Windows 15.0.

Results: Labour induction was successful in $81.33 \%$ of patients. Mean cervical length by digital examination was 2.6 $\mathrm{cm}$, whereas the mean sonographic cervical length was $3.4 \mathrm{~cm}$. There was a significant difference of $0.8 \mathrm{~cm}$ in mean cervical length measured by two methods $(\mathrm{P}=0.01)$. The best cut off point for predicting successful induction of labor was $\leq 3.4 \mathrm{~cm}$ for sonographic cervical length (sensitivity 0.82 , specificity 0.87 , positive predictive value 0.77 and negative predictive value 0.25 ). The best cut off point for Modified Bishop score was 2 (sensitivity 0.95, specificity 0.93). The ROC curve showed that compared to TVS cervical length, Modified Bishop score was the best parameter for predicting successful induction of labor.

Conclusions: Transvaginal sonographic cervical length measurement can be used as an adjunct tool to the traditional Bishop score for predicting successful labor induction in high risk pregnancies.
\end{abstract}

Keywords: Cervical length, Induction of labor, Modified Bishop score, Transvaginal sonography

\section{INTRODUCTION}

Induction of labor is indicated when benefits to the mother or the foetus outweigh those of continuing the pregnancy such as post-dated pregnancy, preeclampsia or foetal growth restriction. It is the most common intervention in modern obstetrics and incidence varies from 13-20\%. ${ }^{1}$ Although induction of labor is safe and efficacious method of vaginal delivery, it still caries considerable risk of caesarean delivery, approximately $20 \%$ compared with spontaneous labor and this risk is significantly influences by the status of the cervix at the time of labor induction. ${ }^{2}$ Therefore, several attempts have been made to assess cervical status prior to induction of labor.

The traditional method of predicting whether an induced labor will result in a successful vaginal delivery is based 
on the digital examination of the cervix. The cervical attributes have been described and quantified into several scoring systems. The most widely accepted is the scoring system described by bishop in $1964 .{ }^{3}$ Five components of the Bishop's score depend solely on the digital assessment of the cervix and the level of presenting part.

One of the most common labor ward problems is the different results of digital assessment of the cervix. The main reason behind this conflict is the subjective nature of digital examination of the cervix, especially the assessment of the cervical length. ${ }^{4}$ Supravaginal portion of the cervix makes up about $50 \%$ of the cervical length and varies from one woman to another. This portion of the cervix is difficult to estimate digitally, and it makes assessment highly subjective. ${ }^{5}$ Digital examination measures only the length from the external cervical os to the cervical-vaginal junction. In addition, examination findings may be affected due to the discomfort associated with the digital assessment of the cervix. ${ }^{6}$ The preinduction 'favourability' of the cervix as assessed by the Bishop score is very subjective, with high inter-observer and intra-observer variation. ${ }^{7}$ Therefore, a reliable and better tolerated method of pre-induction assessment than the bishop score would be a helpful tool in the assessment and counselling of women planned for labor induction.

To assess pre-induction cervical ripeness, transvaginal sonography (TVS) appears to be a feasible alternative to the traditional bishop's score, since it is considered to be reproducible, easy to learn and with images that can be documented for intra and inter-observer comparison. ${ }^{8,9}$ However, it is still controversial whether the ultrasonographic assessment of the cervix is valuable in term or post term gestation for the prediction of successful vaginal delivery. ${ }^{10,11}$ The purpose of this study, was to compare cervical assessment by Transvaginal sonography and digital examination in prediction of outcome of labor induction.

\section{METHODS}

This was a prospective observational study, done in the Department of Obstetrics and Gynecology, Lady Hardinge Medical College and Smt. Sucheta Kriplani Hospital, New Delhi from November 2009 to March 2011. A total of three hundred pregnant women undergoing induction of labour due to some obstetric reason were enrolled in the study after informed written consent. The study received approval of the ethical committee of the hospital.

\section{Inclusion criteria}

- All patients with singleton gestation at 37 completed weeks up to 42 weeks with vertex presentation, longitudinal lie, live fetus, intact amniotic membrane, no vaginal bleeding, reactive non-stress test (NST) and an initial cervical examination showing $\leq 2 \mathrm{~cm}$ dilatation and $\leq 50 \%$ effacement were included in the study.

\section{Exclusion criteria}

- Patients with previous uterine surgery, antepartum hemorrhage, pre-labour rupture of membrane (PROM), major foetal anomalies, contraindication to vaginal delivery and women in active labor were excluded from the study.

After careful history, general and systemic examination, Transvaginal sonography was done to record the cervical length in $\mathrm{cm}$ for each patient.

Before induction of labor, transvaginal ultrasound was performed using Philips HD 3 2D G52/P 90 system with a transvaginal probe of $4-9 \mathrm{MHz}$ patients were asked to empty bladder to avoid a deceptively elongated image caused by full bladder. To avoid any cervical distortion of its position or shape, transducer was placed in the vagina approximately $3 \mathrm{cms}$ proximal to the cervix. Sagittal view of cervix was obtained, with the echogenic endocervical mucosa seen along the length of the endocervical canal. The image was magnified to $75 \%$ of the screen. The length of the cervix was measured from the internal os to the external os, the furthest at which the cervical walls were juxtaposed. Digital examination of the cervix was performed with all aseptic precautions by an obstetrician who was blinded to the ultrasound measurements, and a score was assigned as per Modified Bishop score. ${ }^{12}$ Total Modified bishop score is 10 and score of $0-4$ was taken as unfavourable (Table 1).

Table 1: Modified Bishop's score.

\begin{tabular}{|l|l|l|l|}
\hline \multicolumn{1}{|c|}{ Score } & 0 & 1 & 2 \\
\hline Position of cervix & Posterior & Central & Anterior \\
\hline Length of cervix & $\geq 3 \mathrm{~cm}$ & $1-2 \mathrm{~cm}$ & $\leq 1 \mathrm{~cm}$ \\
\hline Dilatation of cervix & $0 \mathrm{~cm}$ & $1-2 \mathrm{~cm}$ & $3-4 \mathrm{~cm}$ \\
\hline Consistency of cervix & Firm & Medium & Soft \\
\hline Station of head & -3 & -2 & $-1 / 0$ \\
\hline Total score & & & 10 \\
\hline
\end{tabular}

Induction of labor was carried out according to the standard protocol of hospital. If the bishop scores of 4 or less, $0.5 \mathrm{mg}$ Dinoprostone gel was administered intracervical every 6 hours for a maximum of three doses.

This was followed by oxytocin infusion six hours after the last prostaglandin dose as required, if regular uterine contractions and cervical change did not develop. With Bishop's score more than 4, amniotomy followed by oxytocin infusion or oxytocin alone was started.

The patient was closely monitored in labor, for both maternal and foetal wellbeing. For the purpose of this study, successful labor induction was taken as a vaginal delivery within 24 hours from the start of induction. 


\section{Statistical analysis}

Data were analyzed using SPSS for Windows 15.0 . Receiver operating characteristic curves were used to determine the optimal cut-off for total bishop score, sonographic cervical length and digitally measured cervical length.

Univariate analysis was conducted with the student ttest. Multiple logistic regression was used to investigate the usefulness of different parameters in independently predicting successful induction of labor. Statistical significance was considered to be achieved at P-values < 0.05 .

\section{RESULTS}

Almost equal number of primigravida (47.6\%) and multigravida $(52.4 \%)$ were there in the study population. Demographic characteristics of the study population are shown in Table 2.

Table 2: Demographic characteristics of the study population.

\begin{tabular}{|l|l|}
\hline Characteristic & Value \\
\hline $\begin{array}{l}\text { Maternal age, years (Mean } \pm \text { SD, } \\
\text { median, Range) }\end{array}$ & $24.37 \pm 3.2$, \\
\hline Parity, N, (\%) & $24,18-35$ \\
\hline Primigravida & $143(47.6 \%)$ \\
\hline Multigravida & $157(52.4 \%)$ \\
\hline $\begin{array}{l}\text { Gestation age at induction, weeks } \\
\text { (Mean } \pm \text { SD, Median, Range) }\end{array}$ & $39.8 \pm 1.06$, \\
\hline
\end{tabular}

The most common indication for induction of labor was post-dated pregnancy (73\%). The other indications were: gestational hypertension $(9.6 \%)$, foetal growth restriction (8\%), decreased foetal movement (4.6\%), oligohydramnios (3\%), maternal diabetes mellitus $(0.6 \%)$, maternal cholestasis $(0.6 \%)$ and bad obstetric history $(0.3 \%)$.

Vaginal delivery occurred in $260(86.7 \%)$ women. 244 $(93.84 \%)$ of these deliveries was within 24 hours of induction and $16(6.2 \%)$ cases delivered after 24 hours of induction. $40(13.3 \%)$ patients required caesarean section. Out of these 40 patients, 33 patients underwent caesarean section within 24 hours of induction and seven after 24 hours.

Indications for caesarean section were foetal distress $(57.5 \%)$, meconium stained liquor $(25 \%)$, failure to progress $(12.5 \%)$ and failed induction $(5 \%)$. There was no second stage operative vaginal delivery in the study population.

The cervical length $(\mathrm{cm})$ assessed by Transvaginal sonography ranges from $2.4 \mathrm{~cm}$ to $4.5 \mathrm{~cm}$. Mean cervical length was $3.4 \pm 0.42 \mathrm{~cm}$ and median was $3.4 \mathrm{~cm}$ (Table 3 ).
Table 3: Pre-induction cervical length measured by transvaginal sonography.

\begin{tabular}{|l|l|l|}
\hline Cervical length $(\mathrm{cm})$ & No. of cases & $\%$ \\
\hline $2.0-2.5$ & 3 & 1 \\
\hline $2.6-3.0$ & 60 & 20 \\
\hline $3.1-3.5$ & 125 & 41.7 \\
\hline $3.6-4.0$ & 88 & 29.3 \\
\hline $4.1-4.5$ & 24 & 8 \\
\hline Total & 300 & 100 \\
\hline
\end{tabular}

After doing cervical assessment by Transvaginal sonography, digital examination of cervix was done by an Obstetrician blinded to the Transvaginal sonography findings Digital findings of cervix was scored according to modified Bishop score. The cervical length $(\mathrm{cm})$ assessed by digital examination of cervix ranges from $1.5 \mathrm{~cm}$ to $3.5 \mathrm{~cm}$ (Table 4 ).

Table 4: Pre-induction cervical length $(\mathrm{cm})$ measured by digital examination.

\begin{tabular}{|l|l|l|}
\hline Cervical length $(\mathrm{cm})$ & No. of cases & $\%$ \\
\hline $1.5-2.0$ & 8 & 2.7 \\
\hline $2.1-2.5$ & 201 & 67 \\
\hline $2.6-3.0$ & 90 & 30 \\
\hline $3.1-3.5$ & 1 & 0.3 \\
\hline Total & 300 & 100 \\
\hline
\end{tabular}

In women delivered vaginally, mean cervical length measured by TVS was $3.39 \pm 0.422 \mathrm{~cm}$ and by digital examination, mean cervical length was $2.60 \pm 0.30 \mathrm{~cm}$ and was significantly $(\mathrm{P}=0.001)$ shorter than in women in whom caesarean section had to be done. Mean Modified Bishop score of study population was $3.30 \pm 0.71$, Median 3 and Range 0-4. There were 39 women who had low Bishop score (0-2), and 27(69.3\%) of them had vaginal delivery and $12(30.7 \%)$ underwent caesarean section. Women with bishop score between3-4, out of 261 women, $233(89.2 \%)$ had vaginal delivery and 28 (10.8\%) had caesarean section. Modified bishop score was significantly related with mode of delivery $(\mathrm{P}=0.001)$. Maximum number $(56.5 \%)$ of vaginal deliveries occurred within 12 hours of induction. $6.2 \%$ of deliveries occurred in more than 24 hours (Table 5).

Table 5: Induction to delivery interval (hours).

\begin{tabular}{|l|l|l|}
\hline Induction delivery interval & No. of cases & $\%$ \\
\hline Within 12 hours & 147 & 56.5 \\
\hline $12.1-24$ hours & 97 & 37.3 \\
\hline More than 24 hours & 16 & 6.2 \\
\hline Total & 260 & 100 \\
\hline
\end{tabular}

Out of 277 cases where Induction to delivery interval was $\leq 24$ hours, $244(88.08 \%)$ cases delivered vaginally and $33(11.91 \%)$ underwent caesarean section. Out of 23 cases where Induction to delivery interval was $>24$ hours of induction, $16(69.56 \%)$ cases delivered by vaginal route 
and $7(30.43 \%)$ by caesarean section. The mean time duration from induction to active phase was $8.86 \pm 3.93$ hours (median 6 hours, range 6 to 18 hours). The mean time duration from active phase to vaginal delivery was $4.68 \pm 1.86$ hours (median 4.33 hours, range 1.16 to 9.33 hours). The mean time duration from induction to delivery interval was $13.26 \pm 4.98$ hours (median 11 hours, range 7.16 to 27.33 hours).

\section{Relationship of transvaginal sonographic cervical length with outcome of labor}

With increasing sonographic cervical length, there was decrease chance of vaginal delivery and increase chance of caesarean section. Women with cervical length between 2 to $2.5 \mathrm{~cm}, 100 \%$ had delivery by vaginal route while with cervical length between 4.1 to $4.5 \mathrm{~cm}, 79.2 \%$ delivered vaginally and rest $(20.8 \%)$ by caesarean section. Sonographic cervical length was significantly related with mode of delivery $(\mathrm{P}=0.004)$.

With increase in sonographic cervical length, Induction to delivery interval also increased. Women with sonographic cervical length between $2-2.5 \mathrm{~cm}$, all (100\%) of them got delivered within 12 hours of induction and with cervical length between 2.6 to $3.0 \mathrm{~cm}, 87 \%$ got delivered within 12 hours. Women with cervical length between 3.1 to $3.5 \mathrm{~cm}, 66.37 \%$ delivered within 12 hours and between 4.1 to $4.5 \mathrm{~cm}$, only $5.25 \%$ got delivered within 12 hours of induction. There was a statistically significant relation between sonographically measured cervical length and the Induction to delivery interval $(\mathrm{P}=0.001)$.

Women with decrease sonographic cervical length, has shorter Induction to active phase interval. Women with cervical length between $2-2.5 \mathrm{~cm}$, all of them achieve active phase within 6 hours of induction and with cervical length between 2.6 to $3.0 \mathrm{~cm}$, induction to active phase duration was 6 hours in $86.7 \%$ of women. Women with cervical length between 3.1 to $3.5 \mathrm{~cm}, 77.6 \%$ achieve active phase within 6 hours and with a cervical length between 4.1 to $4.5 \mathrm{~cm}$, only $4.1 \%$ achieve active phase within 6 hours of induction. There was significant relationship between sonographically measured cervical length and induction to active phase interval $(\mathrm{P}=0.001)$. There was no significant relationship between sonographically measured cervical length with active phase duration $(\mathrm{P}=0.86)$.

\section{Relationship of digital examination findings with the outcome of labor}

Similar to TVS measurement of cervical length, with increase in digitally measured cervical length, there is decrease chance of vaginal delivery and increase chance of caesarean section. Digital cervical length was significantly related with mode of delivery $(\mathrm{P}=0.02)$. Women with cervical length between 1.5 to $2.0 \mathrm{~cm}, 100 \%$ had delivery by vaginal route while with cervical length between 2.6 to $3.0 \mathrm{~cm}, 77.8 \%$ delivered vaginally and rest $(22.2 \%)$ by caesarean section.

With increase in digitally measured cervical length, Induction to delivery interval also increased. Women with cervical length between $1.5-2.0 \mathrm{~cm}$, all $(100 \%)$ of them got delivered within 12 hours of induction and with length between 2.1 to $2.5 \mathrm{~cm}, 66.29 \%$ got delivered within 12 hours. Women with cervical length between 2.6 to 3.0 $\mathrm{cm}, 27.14 \%$ delivered within 12 hours and between 3.1 to $3.5 \mathrm{~cm}$, none of them got delivered within 12 hours of induction. There was a statistically significant relation between digitally measured cervical length and the Induction to delivery interval $(\mathrm{P}=0.04)$.

Women with decreasing digital cervical length, has shorter Induction to active phase duration. Women with cervical length between $1.5-2.0 \mathrm{~cm}$, all of them achieve active phase within 6 hours of induction and with cervical length between 2.1 to $2.5 \mathrm{~cm}$, induction to active phase duration was 6 hours in $80.63 \%$ of women. Women with cervical length between 2.6 to $3.0 \mathrm{~cm}, 40 \%$ achieve active phase within 6 hours and with a cervical length between 3.1 to $3.5 \mathrm{~cm}$, none of them achieve active phase within 6 hours of induction. There was significant relationship between digitally measured cervical length and induction to active interval $(\mathrm{P}=0.037)$. No significant relationship $(\mathrm{P}=0.97)$ was found between digitally measured cervical length with the mean of active phase to vaginal delivery interval. There was a linear relationship between Modified Bishop score and induction delivery interval. Women with higher bishop Score were more likely to deliver early (Figure 1).

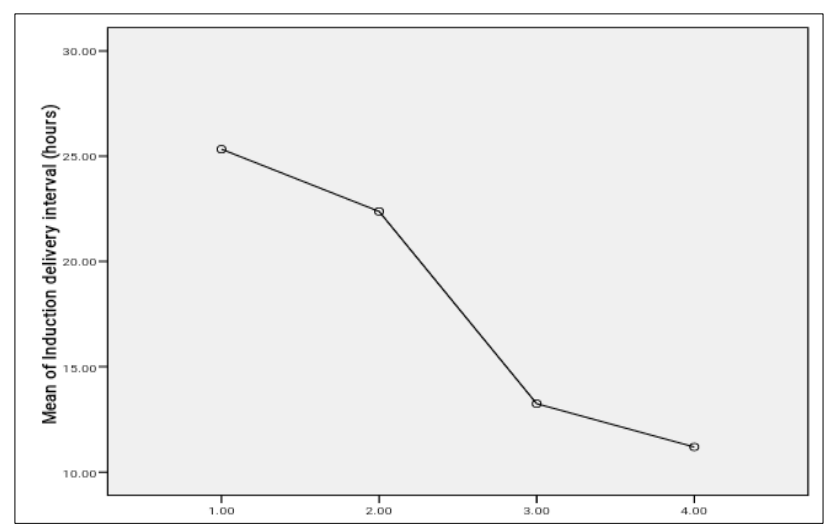

Figure 1: Relationship of modified Bishop score with mean induction to delivery interval (hours).

Women with modified bishop score between 3-4, maximum number $(99.12 \%)$ had vaginal delivery within 24 hours of induction as compared to women with low bishop score (0-2), only $45 \%$ of them had vaginal delivery within 24 hours of induction. Modified bishop score was significantly related with induction delivery interval $(\mathrm{P}=0.004)$. There were 39 women who had low bishop score (0-2), and $27(69.3 \%)$ of them had vaginal delivery and $12(30.7 \%)$ underwent caesarean section. 
Women with Bishop Score between 3-4, out of 261 women, $233(89.2 \%)$ had vaginal delivery and $28(10.8 \%)$ had caesarean section. Vaginal delivery rate was significantly higher $(\mathrm{P}=0.02)$ in the high bishop Score group as compared to the low bishop score group (0-2).

In primigravida, the mean sonographic cervical length was $3.52 \pm 0.68 \mathrm{~cm}$ whereas in multigravida, the mean sonographic cervical length was $3.11 \pm 0.6 \mathrm{~cm}$. The mean difference of sonographic cervical length between primigravida and multigravida was $0.41 \mathrm{~cm}$ and the difference were significant $(\mathrm{P}=0.042)$.

With increasing gravidity there was a favourable bearing on the outcome of labor induction. $97.18 \%$ of multigravida delivered within 24 hours of induction as compared to $89.83 \%$ of primigravida. $10.16 \%$ of primigravida delivered in more than 24 hours of induction as compared to $2.81 \%$ of multigravida. Gravidity was significantly related with Induction to Delivery Interval $(\mathrm{P}=0.02)$. The mean induction to delivery interval in primigravida was $14.28 \pm 5.64$ hours as compared to $11.90 \pm 3.92$ in multigravida. Mean Induction to active phase interval (hours) was 7.32 hours in multigravida as compared to 9.79 hours in primigravida.

There was significant $(\mathrm{P}=0.04)$ relationship between gravidity and mean of induction to active phase interval. Mean duration of active phase to vaginal delivery was 4.89 hours in primigravida as compared to 4.24 hours in multigravida. There was statistically insignificant relationship between gravidity and mean of active phase to vaginal delivery interval $(\mathrm{P}=0.086)$.

\section{Correlation}

Association of sonographically measured cervical length (Figure 2) and digitally measured cervical length (Figure 3) with induction delivery interval showed that sonographically measured cervical length was the better predictor of the induction delivery interval.

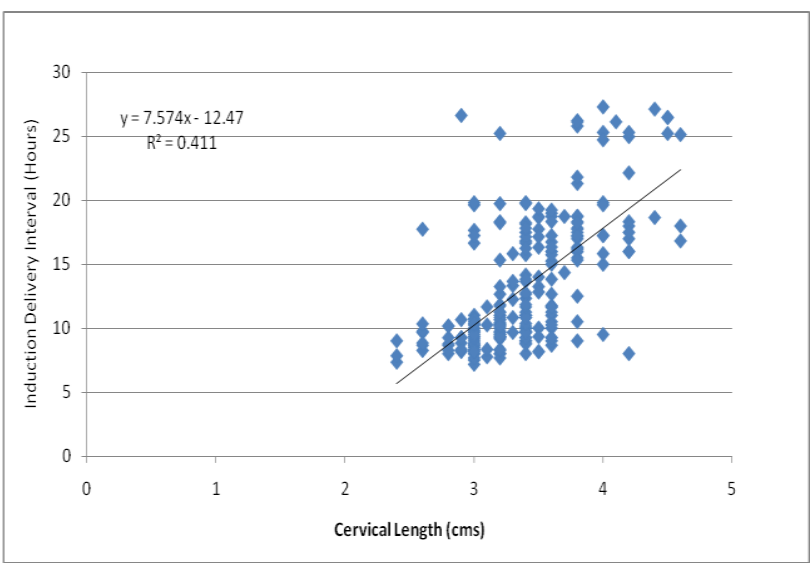

Figure 2: Correlation between pre -induction cervical length $(\mathrm{cm})$ measured sonographically and the induction to delivery interval (hours).
The R2 value for the sonographically measured cervical length was 0.41 compared with the $\mathrm{R} 2$ value of 0.17 for the digitally measured cervical length (Figure 3 ).

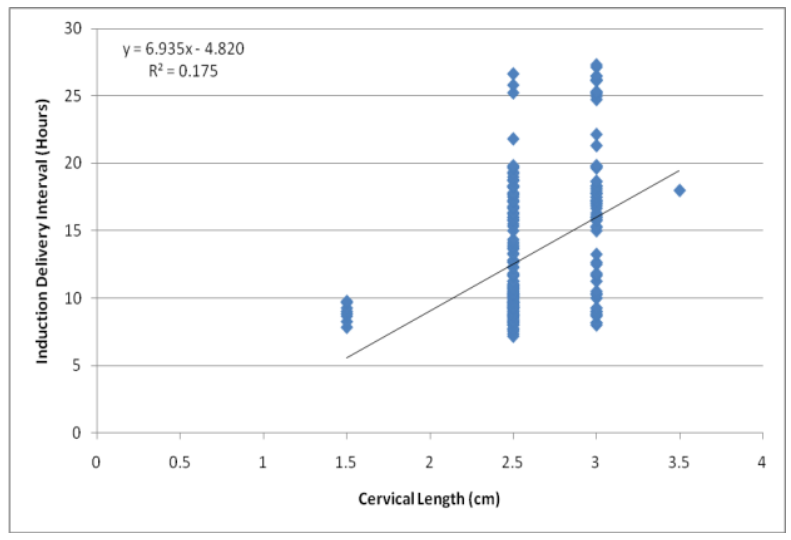

Figure 3: Correlation-association between preinduction cervical length $(\mathrm{cm})$ measured by digital examination and the induction to delivery interval (hours).

Receiver operating characteristic curves (ROC) was constructed to find the best cut-off values of sonographically measured cervical length, digitally measured cervical length and Modified Bishop score for prediction of successful induction of labor. In ROC curves, the best cut off point for predicting successful induction of labor was $\leq 3.4 \mathrm{~cm}$ for cervical length measured by transvaginal sonography (sensitivity 0.82 , specificity0.87, positive predictive value 0.77 and negative predictive value 0.25 ). The likelihood ratio (sensitivity/1-specificity) of this cut-off value was 6.30 . The area under the ROC curve is 0.72 . The curve constructed for sonographically measured cervical length was above the $45^{\circ}$ line, indicating a significant relationship between this variable and successful induction of labor (Figure 4).

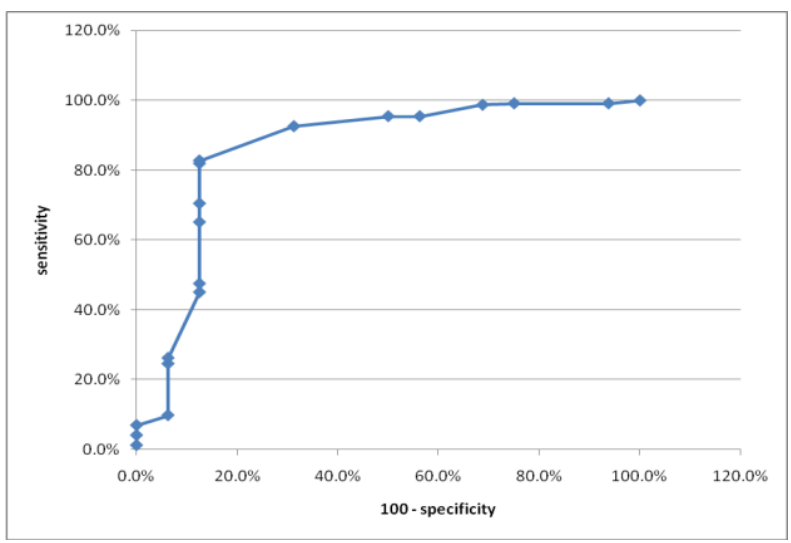

Figure 4: Receiver operating characteristic curve analysis for sonographically measured cervical length in predicting successful induction of labor.

In ROC curve, the best cut off point for digitally measured cervical length for predicting successful 
induction of labor was $2.5 \mathrm{~cm}$ (sensitivity 0.76 , specificity 0.81 , positive predictive value 0.98 and negative predictive value 0.18 ). The likelihood ratio (sensitivity/ 1 -specificity) of this cut-off value was 4 . The area under the ROC curve is 0.58 (Figure 5).

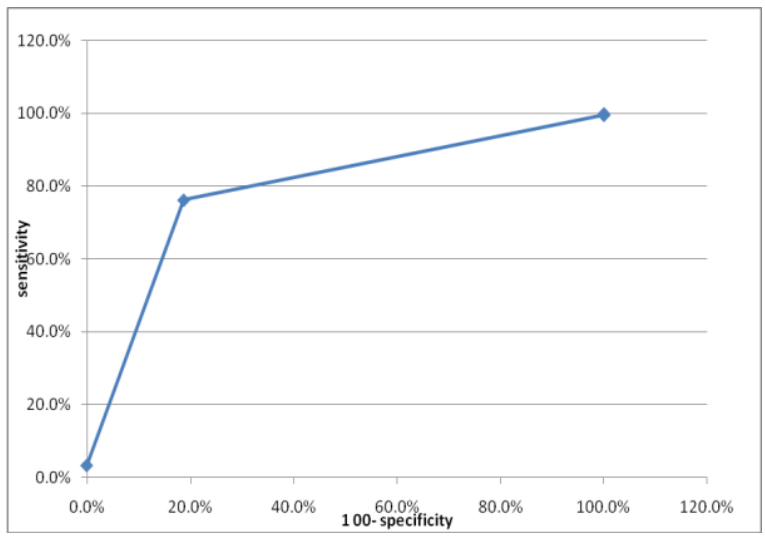

Figure 5: Receiver operating characteristic curve analysis for digitally measured cervical length in predicting successful induction of labor.

In ROC curve, the best cut off point for predicting successful induction of labor was 2 for modified bishop score (sensitivity 0.95 and specificity 0.93 ). The likelihood ratio (sensitivity/ 1-specificity) of this cut-off value was 13.57 . The area under the ROC curve is 0.93 (Figure 6).

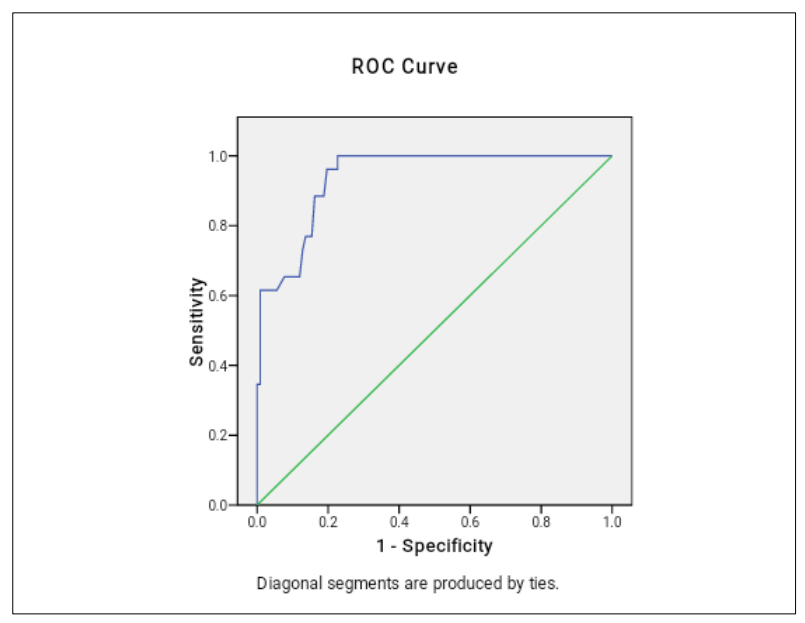

Figure 6: Receiver operating characteristic curve analysis for modified bishop score in predicting successful induction of labor.

Logistic regression was used to look at the individual components of the Bishop's score and sonographically measured cervical length, how well they predict successful induction of labor. Multiple logistic regression analysis shows that among the transvaginal sonographic findings, sonographic cervical length was significantly related to successful labor induction (Pvalue 0.03, OR 1.26, 95\% CI 0.013- 2.96). Modified bishop score was also found to be significantly related with successful labor induction ( $\mathrm{P}$ value $<0.001$, OR 89.76 , 95\% CI 8.694-926.655). Among the individual parameters of bishop score, only cervical consistency was found to be significantly related to successful labor induction ( $\mathrm{P}$ value 0.03 , OR $1.345,95 \%$ CI 0.168 - 0.876). Gravidity and gestational age at the time of induction were not significantly related to successful labor induction.

Multiple logistic regression analysis also showed that among the Modified Bishop score and TVS cervical length, Modified Bishop score is the better predictor of successful labor induction. Among the parameters evaluated in this study, Modified bishop score and transvaginal sonographic cervical length could accurately predict vaginal delivery within 24 hours of induction and were independently associated with labor duration, whereas digitally measured cervical length failed to accurately predict the outcome of induced labor. Our data suggested that the measurement of cervical length by Transvaginal ultrasonography is a useful and independent predictor of successful labor induction and can be used as an adjunct tool to the traditional bishop score. The foetal outcome was good with no stillbirth and early neonatal deaths. All of them had good Apgar score (7-10) at 1 minute and 5 minutes. The mean birth weight $(\mathrm{kg})$ was found to be $2.7 \pm 0.38 \mathrm{~kg}$ (Median $=2.7 \mathrm{~kg}$, Range $=1.6 \mathrm{~kg}$ $3.8 \mathrm{~kg}$ ). There was no maternal complication in the study population.

\section{DISCUSSION}

Many studies have been done regarding the role of Transvaginal ultrasonographic cervical assessment in predicting the outcome of induction of labor. However, it is still controversial whether ultrasonographic assessment of the cervix is valuable for the prediction of successful induction of labor in term gestation or not. Some investigators have reported the TVS assessment of the cervix is of value for predicting the outcome of induction. At the same time, some studies have shown that the Bishop score has a better predictive value associated with the result of outcome of labor induction.

Although Transvaginal ultrasonography has been well recognized as being predictive of impending labor in high-risk patients for preterm delivery, the value of the method for pre-induction cervical assessment in term pregnancy remains controversial. The present study was done to determine the usefulness of transvaginal sonographic cervical measurements in predicting the outcome of induction of labor. The results of this study were similar to a great extent to the result obtained by Elghorori et al, and Selhi $M$ et al regarding the age, gestational age at induction, parity and indications of labor induction. ${ }^{13,14}$ In present study, vaginal delivery occurred in $260(86.7 \%)$ women and 40(13.3\%) women required caesarean section. Our findings are consistent with various studies conducted by Paterson -Brown et al (86\% VD ,14\% LSCS), Elghorori et al $(83.7 \%$ VD, 
$16.3 \%$ LSCS $)$ and Selhi $M$ et al, $(88.34 \%$ VD, $13.3 \%$ LSCS). ${ }^{6,13,14}$ In present study, 244 women out of $300(81.33 \%)$ had successful induction of labor. Our finding of successful induction of labor are consistent with studies done by Chandra et al $(80 \%)$, Rane et al $(80.1 \%)$, Elghorori et al $(83.7 \%)$ and Uyar $\mathrm{Y}$ et al $(83.1 \%) \cdot{ }^{13,15-17}$ Very few studies have reported on duration of labor. Yang et al reported mean duration of induction to active phase as $15.3 \pm 13.1$ hours. ${ }^{18}$ This can be explained by in Yang et al, study percentage of primigravida were more (74\%) as compared to $47.6 \%$ of primigravida in our study. ${ }^{18}$ Yang et al, reported mean duration of active phase as $4.1 \pm 3.16$ hours which is in consistent with our active phase duration. ${ }^{18}$ Yang et al, study did not comment on mean induction to delivery interval. $^{18}$

Mean sonographic cervical length was $3.4 \pm 0.42 \mathrm{~cm}$ in present study. Our findings are consistent with the study conducted by Elghorori et al, which reported mean sonographic cervical length of $3.4 \mathrm{~cm} .{ }^{13}$ In present study, mean cervical length of study population measured by digital examination was $2.6 \mathrm{~cm} \pm 0.29 \mathrm{~cm}$. There was a significant difference of $0.8 \mathrm{~cm}$ in mean cervical length measured by two methods $(\mathrm{P}=0.01)$. The difference in mean cervical length by two methods can be explained by the fact that the supravaginal portion of the cervix makes up about $50 \%$ of the cervical length and varies from one woman to another. This portion of the cervix is difficult to estimate digitally, and it makes assessment highly subjective. Digital examination measures only the length from the external cervical os to the cervical-vaginal junction. In present study, the best cut off point for predicting successful induction of labor was $\leq 3.4 \mathrm{~cm}$ for cervical length measured by Transvaginal sonography (sensitivity 0.82 , specificity 0.87 , positive predictive valueb0.77 and negative predictive value 0.25 ). The likelihood ratio (sensitivity/ 1-specificity) of this cut-off value was 6.30. The area under the ROC curve was 0.72 . Our findings are consistent with study conducted by Yang et al, (Table 6). ${ }^{18}$
In ROC curve, the best cut off point for predicting successful induction of labor was 2 for modified bishop score (sensitivity 0.95 and specificity 0.93). The likelihood ratio (sensitivity/ 1-specificity) of this cut-off value was 13.57. The area under the ROC curve was 0.93 . Whereas in previous studies, the cut-off value of bishop score was higher as compared to present study. The reason for this discrepancy is that, previous studies used bishop score for assessing favorability for induction of labor and total bishop score ranged from 0 to 13 .

In present study, we used modified bishop score. This scoring system gave each of Bishop's categories a maximum score of 2, for a total maximum score of 10 . Modified bishop score considered effacement in terms of centimeter in length as opposed to percentage effaced. The ROC curve of present study showed that compared to TVS cervical length, Modified bishop score was the best parameter for predicting successful induction of labor (area under ROC curve of modified bishop score was more than the TVS cervical length). Univariate analysis was done to analyze the individual parameters of Transvaginal sonography, modified bishop score and maternal demographic characteristics for predicting successful induction of labor. In present study, among the transvaginal ultrasonographic cervical findings successful induction of labor was significantly related with sonographic cervical length $(\mathrm{P}<0.001)$, Modified Bishop score $(\mathrm{P}<0.001)$ was also found to have significant relationship with successful labor induction. Successful induction of labor was significantly related to maternal age $(\mathrm{P}=0.002)$, and gravidity $(\mathrm{P}=0.02)$. Gestational age at time of induction $(\mathrm{P}=0.116)$ was not significantly related to successful induction of labor. In present study, multiple logistic regression analysis showed that among the modified Bishop score and TVS cervical length, Modified bishop score is the better predictor of successful labor induction. There were many studies done regarding the role of pre-induction cervical assessment in predicting successful labor induction (Table 6).

Table 6: ROC analysis of TVS cervical length and bishop score: comparison of various studies.

\begin{tabular}{|c|c|c|c|c|c|c|c|c|}
\hline Author & & TVS & rvical lengt & & & & op score & \\
\hline & Cut- off & $\mathbf{A U C}$ & Sensitivity & Specificity & Cut -off & AUC & Sensitivity & Specificity \\
\hline Paterson-Brown et $\mathrm{al}^{6}$ & NR & NR & NR & NR & NR & NR & NR & NR \\
\hline Reis et al $^{10}$ & NR & NR & NR & NR & NR & NR & NR & NR \\
\hline Rane et al $^{13}$ & $2.4 \mathrm{~cm}$ & 0.82 & $84 \%$ & $59 \%$ & NR & NR & NR & NR \\
\hline Yang et $\mathrm{al}^{18}$ & $3.0 \mathrm{~cm}$ & 0.77 & $75 \%$ & $83 \%$ & 4 & 0.69 & $51 \%$ & $75 \%$ \\
\hline Elghorori et al ${ }^{13}$ & $\leq 3.4 \mathrm{~cm}$ & 0.84 & $62 \%$ & $100 \%$ & $>5$ & 0.5 & $23 \%$ & $88 \%$ \\
\hline Park KH ${ }^{11}$ & $2.8 \mathrm{~cm}$ & 0.67 & $62 \%$ & $60 \%$ & NR & NR & NR & NR \\
\hline Keepanasseril et $\mathrm{al}^{24}$ & $3.0 \mathrm{~cm}$ & 0.926 & $84.9 \%$ & $90.6 \%$ & 3 & 0.657 & NR & NR \\
\hline Uyar Y et al ${ }^{17}$ & $1.9 \mathrm{~cm}$ & 0.819 & $100 \%$ & $19 \%$ & NR & 0.416 & NR & NR \\
\hline Present study & $\leq 3.4 \mathrm{~cm}$ & 0.72 & $82 \%$ & $87 \%$ & 2 & 0.93 & $95 \%$ & $93 \%$ \\
\hline
\end{tabular}


From the previous studies done on prediction of successful labor induction, Paterson-Brown et al, Chandra et al and Reis et al reported bishop score as a better predictor than transvaginal ultrasonographic assessment of cervical length. ${ }^{6,10,15}$ According to Gonen et al and Bueno et al studies, both Bishop score and TVS cervical length shows similar result to predict the successful labor induction. ${ }^{19,20}$ Previous studies done by Ware and Raynor et al, Gabriel et al, Rane et al, Daskalakis et al, Elghorori et al and Keepanasseril A et al, reported transvaginal ultrasonographic cervical assessment as better predictor than bishop score for predicting successful labor induction. ${ }^{13,16,21-24}$

\section{CONCLUSION}

Present study concluded that, among the parameters evaluated, both modified bishop score and transvaginal sonographic cervical length could accurately predict successful induction of labor within 24 hours of induction. On doing regression analysis, modified bishop score was the better predictor of successful labor induction as compared to TVS cervical length. The limitations of our study are that we did not include the other parameters of the transvaginal cervical assessment like posterior cervical angle and occipital position which could have probably added in the predictability. Though, modified bishop score is the better predictor of successful labor induction. Our data suggested that the measurement of cervical length by transvaginal ultrasonography is also a useful and independent predictor of successful labor induction. Therefore, more liberal application of Transvaginal ultrasonography for pre-induction cervical assessment would enable obstetricians to predict the outcome of labor induction especially in high risk pregnancy. Transvaginal sonographic cervical length measurement can be used as an adjunct tool to the traditional Bishop score for predicting successful labor induction in high risk pregnancy.

Funding: No funding sources

Conflict of interest: None declared

Ethical approval: The study was approved by the Institutional Ethics Committee

\section{REFERENCES}

1. Zhang J, Yancey MK, Henderson CE. U.S. National trends in labor induction.1989-1998. J Reprod Med. 2002;47:120-24.

2. Sharma SK, Nagpal M, Thukral CL. Evaluation of pre induction scoring by clinical examination vs transvaginal sonography. Int J Reprod Contracept Obstet Gynecol. 2017;6:228-31.

3. Bishop EH. Pelvic scoring for elective induction. Obstet Gynecol. 1964;24:266-8.

4. Bansiwal R, Rao R, Mishra N, Kapur V. Bishop score and transvaginal ultrasound for preinduction cervical assessment: a randomized clinical trial. Int J
Reprod Contracept Obstet Gynaecol. 2013;2(4):6115.

5. Buyer J, Papiernik E, Dreyfus J, Collins D, Wninisdoerffer B, Gueguen S. Maturation signs of the cervix and prediction of preterm birth. Obstet Gynecol. 1986;68:209-14.

6. Paterson-Brown S, Fisk NM, Edmonds DK, Rodeck $\mathrm{CH}$. Preinduction cervical assessment by Bishop's score and transvaginal ultrasound. Eur $\mathrm{J}$ Obstet Gynecol Reprod Biol. 1991;40:17-23.

7. Goldberg J, Newman RB, Rust PF. Interobserver reliability of digital and endovaginal ultrasonographic measurements. Am J Obstet Gynecol. 1997; 177:853-8.

8. Lazanakis M, Marsh MS, Brockbank E, Economides DL. Assessment of the cervix in the third trimester of pregnancy using transvaginal ultrasound scanning. Eur J Obstet Gynecol Reprod Biol. 2002;105:31-5.

9. Anderson HF. Transvaginal and transabdominal ultrasonography of the uterine cervix during pregnancy. Am J Clin Ultrasound. 1991;19(2):77-83.

10. Reis FM, Gervasi MT, Florio P. Prediction of successful induction of labor at term: Role of clinical history, digital examination, ultrasound assessment of the cervix, and fetal fibronectin assay. Am J Obstet Gynecol. 2003;189:1361-67.

11. Park KH. Transvaginal ultrasonographic cervical measurement in predicting failed labour induction and caesarean delivery for failure to progress in nulliparous women. J Korean Med Sci. 2007;22:7227.

12. Burnett JE. Preinduction scoring: An objective approach to induction of labor. Obstet Gynecol. 1996;28:479-83.

13. Elghorori MR, Hassan I, Dartey W, Abdel-Aziz E, Bradley M. Comparison between subjective and objective assessment of cervix before induction of labor. Obstet Gynecol. 2006;26(6):521-6.

14. Selhi M, Surapeni T. Preinductionsonographic measurement of cervical length: An adjunct to Bishop score? Amithyaa- Fernandez Hospital. 2010;1(2).

15. Chandra S, Crane JM, Hutchens D, Young DC. Transvaginal ultrasound and digital examination in predicting successful labor induction. Obstet Gynecol. 2001;98:2-6.

16. Rane SM, Pandis GK, Guirgis RR, Higgins B, Nicolaides KH. Preinductionsonographic measurement of cervical length in the prolonged pregnancy: the effect of parity in the prediction of induction to delivery interval. J Ultrasound in Obstet Gynaecol. 2003;22:40-4.

17. Uyar Y, Erbay G, Demir BG, Baytur Y. Comparison of the Bishop score, body mass index and transvaginal Cervical length in predicting the success of labor induction. Arch Gynaecol Obstet. 2009;280:357-62.

18. Yang SH, Roch CR, Kim JH. Transvaginal ultrasonography for cervical assessment before 
induction of labour. Am J Ultrasound Med. 2004;23:375-82.

19. Gonen R, Degani S, Ron A. Prediction of successful induction of labour: Comparison of transvaginal ultrasonography and Bishop score. Eur J Ultrasound 1998;7:183-7.

20. Bueno B, San Frutos L, Salazar F, Perez-Medina T, Engels V, Archilla B, et al. Variables that predict the success of labor induction. Acta Obstet Gynecol Scand. 2005;84:1093-97.

21. Ware V, Raynor BD. Transvaginal ultrasonographic cervical measurement as a predictor of successful labor induction. Am J Obstet and Gynecol. 2000;182:1030-32.

22. Gabriel R, Darnaud T, Chalot F, Gonzalez N, Leymarie F, Quereux C. Transvaginal sonography of the uterine cervix to labour induction. J Ultrasound Obstet Gynaecol. 2002;19:254-7.
23. Daskalakis G, Thomakos N, Hatziioannou L, Mesogitis S, Papantoniou N, Antasaklis A. Sonographic cervical length measurement before labour induction in term nulliparous women. J Foetal Diagn There. 2006;21:34-8.

24. Keepanasseril A, Suri V, Bagga R, Aggarwal N. Preinduction sonographic assessment of the cervix in the prediction of successful induction of labor in nulliparous women. Austral NZ J Obstet Gynaecol. 2007;47:389-93.

Cite this article as: Aggarwal K, Yadav A. Role of transvaginal ultrasonographic cervical assessment in predicting the outcome of induction of labor. Int $\mathbf{J}$ Reprod Contracept Obstet Gynecol 2019;8:628-36. 